\title{
DIAGNÓSTICO MATEMÁTICO DE LA MONITORÍA FETAL CON LA LEY DE ZIPF-MANDELBROT Y LA TEORÍA DE LOS SISTEMAS DINÁMICOS APLICADOS A LA FISIOLOGÍA CARDIACA
}

\author{
Mathematical diagnosis of fetal monitoring \\ using the Zipf-Mandellbrot law and dynamic \\ systems' theory applied to cardiac physiology \\ Javier Rodríguez, M.D.*, Signed Prieto**, Liliana Ortiz***, Alejandro Bautista, \\ M.D.****, Luisa Álvarez ${ }^{+}$, Catalina Correa ${ }^{++}$, Nicolás Avilán ${ }^{+++}$ \\ Recibido: diciembre 15/05 - Revisado: mayo 25/06 - Aceptado: junio 7/06
}

\section{RESUMEN}

Objetivo: desarrollar un diagnóstico matemático de la monitoría fetal a partir de la aplicación de la ley de Zipf-Mandelbrot y las concepciones de salud y enfermedad de los sistemas dinámicos a la aparición de Componentes Dinámicos del Sistema (CDS) en el trazado de la monitoría. Esta evaluación se hace calculando el grado de complejidad de la distribución de los CDS.

Diseño: este estudio es de concordancia diagnóstica basado en una aplicación de la ley de los lenguajes naturales y de simplificaciones fisiológicas basadas en la teoría de sistemas dinámicos a la aparición

\footnotetext{
Correo electrónico: grupoinsight2025@yahoo.es

Grupo Insight

Instituto Materno Infantil

Universidad Nacional de Colombia

* Médico investigador, Director Grupo Insight, profesor Facultad de medicina, Universidad Nacional de Colombia (Sede Bogotá).

** Investigadora Grupo Insight

*** Investigadora Grupo Insight. Delineante de arquitectura.

**** Médico Ginecoobstetra, Universidad Nacional de Colombia (Sede Bogotá) Facultad de medicina, Instituto Materno Infantil.

$+\quad$ Psicóloga, Investigadora Grupo Insight

++ Psicóloga, Investigadora Grupo Insight

++ Físico, Investigador Grupo Insight
}

de CDS de las frecuencias cardiacas fetales para construir una generalización diagnóstica

Materiales y métodos: se evaluaron 100 monitorías de mujeres gestantes divididas en dos grupos: grupo A, 50 gestantes con factores de riesgo, y grupo B, 50 sin factores de riesgo. Basados en simplificaciones dinámicas, prototipos seleccionados y en la aplicación de la ley de Zipf-Mandelbrot para caracterizar el grado de complejidad usando todos los CDS posibles, se realizaron comparaciones con el resto de las monitorías diferenciando salud de enfermedad.

Resultados: la dinámica cardiaca de un feto sano tiene una autorganización matemática caracterizada por su grado de complejidad y la ausencia de CDS Invertidos Pronunciados (CDSiP) y la enfermedad es caracterizada por la pérdida de complejidad o la presencia de uno o más CDSiP, o la presencia de un CDS Invertido (CDSi) mayor o igual a 20x40 hasta 20x50 asociado a la aparición de otro CDSi mayor o igual de 20x50 o de la ausencia de CDS del grupo de 15 y/o de 20 latidos/minuto de altura, o combi- 
naciones de las medidas diagnósticas. De acuerdo a las medidas obtenidas una de cada dos monitorías del grupo B y una de cada seis del grupo A tienen un diagnóstico equivocado según los parámetros clínicos convencionales.

Conclusiones: la caracterización matemática de las monitorías permitió diferenciar salud de enfermedad de manera objetiva y reproducible en el desarrollo de un diagnóstico de aplicación clínica.

Palabras clave: bienestar fetal, monitoreo fetal, ley de Zipf-Mandelbrot, sistemas dinámicos.

\section{SUMMARY}

Objective: developing a mathematical approach to fetal heart rate monitoring by applying ZipfMandelbrot law and 17 conceptions of dynamic systems' health and illness to the appearance of Dynamical Components of the System (DCS) in the monitoring trace by evaluating the degree of complexity of DCS distribution.

Design: this is a diagnostic concordance study based on applying the law of natural languages and physiological simplifications (based on the dynamical systems theory) to the DCS appearance of fetal heart rate (FHR) DCS for making diagnostic generalization.

Material and methods: a set of one hundred monitoring tests from pregnant patients was evaluated and divided into two groups; group A consisted of 50 women having risk factors and group B by 50 women without them. Dynamic simplifications, selected prototypes and ZipfMandelbrot law (for characterizing the degree of complexity using all possible DCSs) were compared with the rest of the monitoring traces to differentiate between health and illness.

Results: a healthy fetus' cardiac dynamics have mathematical self-organization, characterized by its degree of complexity and the absence of pronounced inverted Dynamical Components of the System (piDCS). The disease is characterized by the lack of complexity, or the presence of one or more
piDCS, or the presence of an inverted DSC (iDSC) equal to or higher than $20 \times 40$ associated with the appearance of other iDCS equal to or higher than 20x50, or by the lack of the group of DCSs having 15 and/or 20 beats per minute, or combinations of the diagnostic measurements. One out of two monitoring tests from group B and one out of six from group A returned an incorrect diagnosis (using conventional clinical parameters) according to the computed measurements.

Conclusions: mathematical characterization of the monitoring traces led to objectively and reproducibly differentiating between health and illness in developing a clinical diagnosis.

Key-words: fetal well-being, fetal monitoring, ZipfMandelbrot law, dynamic system components.

\section{INTRODUCCIÓN}

La geometría fractal desarrollada por Benoît Mandelbrot ${ }^{1-3}$ permite caracterizar objetos irregulares determinando su dimensión fractal. ${ }^{4,5}$ Los objetos naturales se consideran fractales salvajes (wild fractals), estos objetos presentan superposición entre sus partes y su dimensión fractal puede ser calculada con el método de Box-Counting, ${ }^{5}$ el cual se ha aplicado en el cuerpo humano a diferentes escalas incluyendo redes vasculares sanas y cancerígenas. ${ }^{6,7}$ Además ha sido útil en modelos de experimentación animal con arterias coronarias, donde se diferenciaron matemáticamente arterias sanas de enfermas evaluando intervenciones moleculares. ${ }^{8}$

Otro tipo de fractal está expresado en la ley de Zipf-Mandelbrot, ${ }^{9-11}$ una ley empírica que permite estudiar cualquier fenómeno que se pueda describir mediante la aparición de frecuencias de alguna variable. Zipf desarrolló una organización jerárquica para las frecuencias de aparición de palabras en un texto, asignando rangos a estas frecuencias y haciendo corresponder a la mayor frecuencia el menor rango, aumentando en forma inversamente proporcional a las frecuencias lo que muestra un comportamiento hiperbólico del fenómeno. Mandelbrot definió la 
dimensión fractal estadística a partir de una linealización logarítmica de las frecuencias y de los rangos calculando el inverso de la pendiente de esta linealización. Esta dimensión fractal fue usada para evidenciar la pérdida de la complejidad de los textos de cartas escritas por monjas que padecían el mal de Alzheimer. ${ }^{12}$ Es el único método que calcula la autoorganización y la coherencia del sistema con dimensiones fractales.

A partir de una analogía con la ley de los lenguajes naturales en el repertorio inmune $\mathrm{B}$ (contra la hemaglutinina A pr8 del virus de la influenza) y el repertorio de linfocitos $\mathrm{T}$ (citotóxicos sanos) se aplicó la ley de Zipf-Mandelbrot. En esta analogía, las reacciones positivas y negativas del repertorio representan las letras, las frecuencias de aparición de linfocitos especializados juegan el papel de las palabras en un texto y el repertorio inmune juega el papel del discurso. Con este análisis se mostró que el repertorio inmune presenta un comportamiento fractal estadístico, en comparación con la respuesta inmune $\mathrm{T}$ de ratones irradiados y ratones atímicos. ${ }^{13,14}$

Recientemente Rodríguez ${ }^{15}$ calculó la dimensión fractal del repertorio inmune $\mathrm{T}$ y clones de células Th contra un alergeno específico, el Poa P9, de un paciente alérgico (en presencia y ausencia de interferón alfa) evidenciando el comportamiento fractal de estos repertorios y el patrón Th y midiendo de forma objetiva y reproducible en inmunoterapias, además de encontrar correlaciones clínicas y matemáticas respecto a la salud y a la enfermedad.

Ahora bien, la teoría de los sistemas dinámicos estudia el estado y evolución de los sistemas partiendo de la definición de variables de estado y describiendo las leyes matemáticas que gobiernan el comportamiento de las mismas. La dinámica del sistema se puede representar con el comportamiento de las variables de estado en el espacio de fases, cuyo comportamiento puede ser descrito por tres tipos de atractores para cualquier sistema: el punto, el ciclo y el atractor caótico; los dos primeros predecibles y el último impredecible. ${ }^{6,16}$ Goldberger, basado en la teoría de los sistemas dinámicos aplicada a la fisiología cardiaca asoció a cada atractor el estado de salud o enfermedad: afirmó que un sistema sano es representado por un atractor caótico, mientras que los sistemas enfermos presentan tendencia a ciclos y a comportamientos periódicos. ${ }^{6}$ Posteriormente, evaluando frecuencias cardiacas en el tiempo y correlacionándolas con diagnósticos clínicos, planteó que los sistemas enfermos se caracterizan por presentar alta periodicidad o excesiva aleatoriedad siendo el comportamiento intermedio de dichos extremos característica de la salud. ${ }^{17}$

Recientemente, basados en estas concepciones de los sistemas dinámicos Rodríguez y colaboradores $^{18}$ desarrollaron una evaluación, con la teoría de la probabilidad aplicada a cuatro CDS. Se partió de cuatro monitorías básicas escogidas con una reinterpretación de la concepción salud enfermedad de los sistemas dinámicos, las cuales fueron comparadas con otros 46. De acuerdo a esta metodología se encontraron 420 posibles monitorías logrando caracterizaciones matemáticas objetivas, las cuales constituyeron un primer paso para diferenciar salud y enfermedad en la monitoría fetal.

En este trabajo, la ley de Zipf-Mandelbrot se aplicó a la evaluación de la aparición de cambios en la frecuencia cardiaca fetal agrupados en CDS. Para esto se desarrolló una analogía lingüística en donde los CDS escogidos reemplazan las palabras y la totalidad de estos el discurso (esto para cada monitoría particular). El total de las monitorías fue evaluado en el contexto de cuatro monitorías seleccionadas con una reinterpretación de la concepción salud enfermedad de los sistemas dinámicos y aplicada a las otras 96 monitorías, en el desarrollo de un diagnóstico objetivo reproducible y de aplicación clínica. En la actualidad no existe un diagnóstico reproducible de la monitoría fetal que supere los problemas intra e inter observador, ${ }^{19}$ el objetivo de este estudio es la aplicación de la ley de ZipfMandelbrot y la reinterpretación de sistemas dinámicos para desarrollar una metodología diagnóstica de aplicación clínica. 


\section{MATERIALES Y MÉTODOS}

Se evaluaron 100 registros tomados con monitor fetal, marca Corometrics ${ }^{\circledR}$. Estos monitores registran la frecuencia cardiaca fetal con una velocidad de 3 centímetros por minuto y una escala de 30 latidos por minuto por cada centímetro de papel. Se evaluaron 50 monitorías de fetos con factores de riesgo, grupo A y 50 sin factores de riesgo, grupo B, con edad gestacional mayor de 28 semanas. Las monitorías del grupo A fueron tomados de gestantes que ingresaron al servicio del Instituto Materno Infantil y las del grupo B del Hospital de Engativá.

Los grupos fueron hechos para poder discernir si los factores de riesgo son importantes o no respecto a la evaluación matemática que se está haciendo.

Cuando una monitoría presenta distribuciones de muy pocas frecuencias cardiacas la dimensión fractal tendrá valores de cero o tendientes a cero por lo tanto se esperan valores que diferencien salud de enfermedad con la aplicación de la ley.

Basados en una reinterpretación propia de concepciones y resultados de la teoría de los sistemas dinámicos, se seleccionaron los cuatro prototipos fundamentales, dos monitorías normales y dos enfermos según diagnóstico clínico convencional sin dudas, uno prácticamente plano y otro con desaceleraciones pronunciadas diagnosticadas clínicamente como sufrimiento fetal agudo. Se evaluó la aparición de CDS en los prototipos y luego se compararon con cualquier otra monitoría siendo esta simplificación y esta comparación el fundamento del desarrollo diagnóstico.

Para la medición de CDS se construyó una rejilla de $6 \mathrm{~cm}$ de base por $5 \mathrm{~cm}$ de altura, la cual contiene los CDS totales. La rejilla tiene divisiones horizontales cada 5 latidos/minuto y verticales cada 5 segundos, ésta es aplicada a cada monitoría llevándola a través del registro de izquierda a derecha, midiendo los CDS que describen aumentos y disminuciones en el sentido en el que va el trazado de la monitoría, midiendo el CDS más grande que esté contenido en determinada variación de la frecuencia cardiaca. Para calcular el número total de apariciones de los
CDS se cuenta una sola vez los CDS más pequeños dentro de los grandes, evaluando todos los aumentos y descensos de forma consecutiva en el trazado; sin tomar en cuenta las interrupciones. Si no se presentan cambios en las alturas de la frecuencia cardiaca no se mide ningún CDS.

Los grupos de CDS que más se presentan serán seleccionados para la evaluación con la ley de Zipf-Mandelbrot y obtener la dimensión fractal de la dinámica cardiaca fetal de acuerdo con una analogía lingüística para cada monitoría particular, en donde los CDS evaluados representan las palabras y la totalidad de estos, el discurso. Siguiendo la metodología de la ley Zipf-Mandelbrot ${ }^{15}$ inicialmente se obtiene el comportamiento hiperbólico para las distribuciones de frecuencias de CDS asignando a mayor frecuencia menor rango y construyendo la respectiva gráfica, para posteriormente realizar la linealización logarítmica llevada a cabo mediante la aplicación del método de mínimos cuadrados, ${ }^{20}$ para luego hallar el inverso multiplicativo negativo de la pendiente, el cual es la dimensión fractal.

Para la evaluación diagnóstica de cada monitoría fetal se consideran simultáneamente las siguientes medidas:

1. Se compara el número de CDS en cada grupo para las cuatro monitorías fundamentales entre sí y con la totalidad de monitorías buscando diferencias matemáticas entre salud y enfermedad. Para esta comparación se calcula la suma de cantidad de CDS en los grupos de altura mayor que 5 latidos/minuto.

2. Se comparan las dimensiones fractales del grupo de CDS que más se presenta en las cuatro monitorías fundamentales para compararlas posteriormente con la totalidad de monitorías, buscando diferencias matemáticas entre salud y enfermedad.

3. Por último se establecen comparaciones entre las medidas realizadas de los CDS invertidos, comparando fundamentalmente los CDSiP en las cuatro monitorías fundamentales entre sí para posteriormente compararlas con la to- 
talidad de monitorías, buscando diferencias matemáticas entre salud y enfermedad y de esta manera evidenciar matemáticamente el sufrimiento fetal agudo.

\section{Definiciones}

Patrón de oro físico y matemático de saludenfermedad: Se establecieron cuatro prototipos: P1 con ausencia de CDS de 15 y 20 latidos/minuto y $\mathrm{P} 2$ con presencia de CDSiP que son los prototipos para enfermedad. P3 y P4 con presencia de CDS de 15 y 20 latidos/minuto y ausencia de CDSiP que son los prototipos para normalidad.

Componentes Dinámicos del Sistema (CDS)

Totales: Son los cambios de la frecuencia cardiaca fetal durante un período de tiempo, en intervalos de cinco segundos o sus múltiplos (que representa la base del CDS) por intervalos de frecuencias de cinco latidos/minuto o múltiplos de esta altura; se consideran todas las posibilidades de base y altura para obtener cada componente dinámico. Las variaciones en la frecuencia cardiaca pueden ser positivas o negativas según se dé un aumento o una disminución. Cuando no hay altura en latidos/minuto no hay CDS. En adelante para la notación de los CDS se utilizará su medida de base y longitud; por ejemplo, para referirse un CDS de base de 30 segundos y de 25 latidos/minuto se notará 30x25.

CDS invertido (CDSi): Es un CDS cuya variación en la frecuencia es negativa, es decir representa la variación que hay en una disminución de la frecuencia cardiaca fetal.

CDSi pronunciado (CDSiP): Es un CDSi cuyas dimensiones son mayores a 20 segundos de tiempo y 50 latidos por minuto de altura (20x50).

Grupo de CDS: Subconjunto de los CDS totales. Conjunto de CDS medidos con igual altura en latidos/minuto y diferentes bases.

Ley de Zipf-Mandelbrot en los lenguajes naturales: ${ }^{10,11}$ En un texto coherente la frecuencia de aparición de palabras es ordenada por una distribución hiperbólica. Dicha distribución puede ser ex- presada como una ley de potencias, la cual permite un análisis matemático que facilita la evaluación de la complejidad del texto con la dimensión fractal estadística. Para la monitoría fetal las frecuencias de palabras son reemplazadas por los CDS escogidos y el discurso por la totalidad de estos para cada monitoría fetal particular.

Dimensión fractal estadística: ${ }^{13,14}$ Medida numérica del grado de complejidad de un sistema, ésta toma valores reales entre 0 y 1 . A partir de la linealización logarítmica de la ley de potencias, la dimensión fractal estadística se calcula con la ecuación:

$$
D=\frac{\log (\sigma+V)}{\log (F / P)}
$$

donde $\sigma$ es el rango asumido para cada frecuencia (propio de la metodología de la ley de ZipfMandelbrot), $\mathrm{P}$ es la frecuencia de aparición de cada CDS en el trazado de la monitoría, V está definida por $\mathrm{V}=1 / \mathrm{N}-1$ donde $\mathrm{N}$ es el número de frecuencias medidas y $\mathrm{F}$ corresponde a un cofactor secundario en el proceso de linealización. La linealización es realizada aplicando el método de mínimos cuadrados.

\section{Análisis matemático}

Se analizan las dimensiones fractales de acuerdo a la analogía lingüística planteada estableciendo el significado del valor de la dimensión fractal igual a cero, entre 0 y 1 , y mayor que 1 , de acuerdo a la interpretación de la ley de los lenguajes naturales aplicada a los prototipos fundamentales. El mismo análisis se efectúa luego con el resto de las monitorías para encontrar las relaciones de salud-enfermedad.

Para la aplicación de la ley de Zipf-Mandelbrot se requiere una distribución hiperbólica, en caso de presentarse tres tipos de CDS o menos no es posible evaluar su grado de complejidad con ésta metodología, por ello a éstas monitorías se les asigna dimensión fractal de valor cero. 
Después de evaluar simultáneamente la aparición de CDS en todos los grupos se hacen comparaciones con la totalidad de las monitorías estableciendo relaciones de igualdad y/o diferencia en las sumatorias del número de aparición de CDS diferentes del grupo de altura cinco (en el desarrollo del diagnóstico) y finalmente se establecen comparaciones entre el diagnóstico convencional y el desarrollado para cada monitoría.

La concepción simplificadora de la dinámica cardiaca fetal física y matemática planteada y la metodología generalista de evaluación diagnóstica propuesta permiten aplicarse a cualquier monitoría particular sin importar poblaciones ni muestras, por lo que no necesitamos validaciones ni metodologías estadísticas.

\section{RESULTADOS}

Se desarrollaron 1.440 posibles CDS totales, 720 aumentos y 720 disminuciones en la frecuencia cardiaca fetal de los cuales se midieron 79 CDS con aumento de frecuencia y 79 con disminución que se presentaron entre 0 y 78 veces. En total se hallaron 158 variedades de CDS para caracterizar cualquier monitoría, de las cuales se escogió el grupo de altura 5 para el análisis con la ley de Zipf-Mandelbrot. Los valores de las dimensiones fractales estadísticas de las monitorías estuvieron entre 0 y 0,8669 para las monitorías del grupo A y entre 0,495 y 1,585 para el grupo sin factores de riesgo y se presentaron grados de complejidad mayores que una en diez monitorías del grupo B.

La dinámica cardiaca de un feto sano se caracteriza por la ausencia de CDSiP además de la existencia de un grado de complejidad autoorganizado. Mientras que para un feto enfermo se presenta pérdida de la complejidad, también se presenta mínimo un CDSiP, o dos CDSi: uno que este entre $20 \times 40$ y 20x50 y otro mayor o igual a 20x50, o la ausencia de sumas de CDS del grupo de altura 15 y/o de 20 latidos/minuto o asociadas a las medidas anteriores. (Tablas 1 y 2).

Tabla 1. Prototipos con las monitorías fundamentales y su dimensión fractal (DF) asociada.

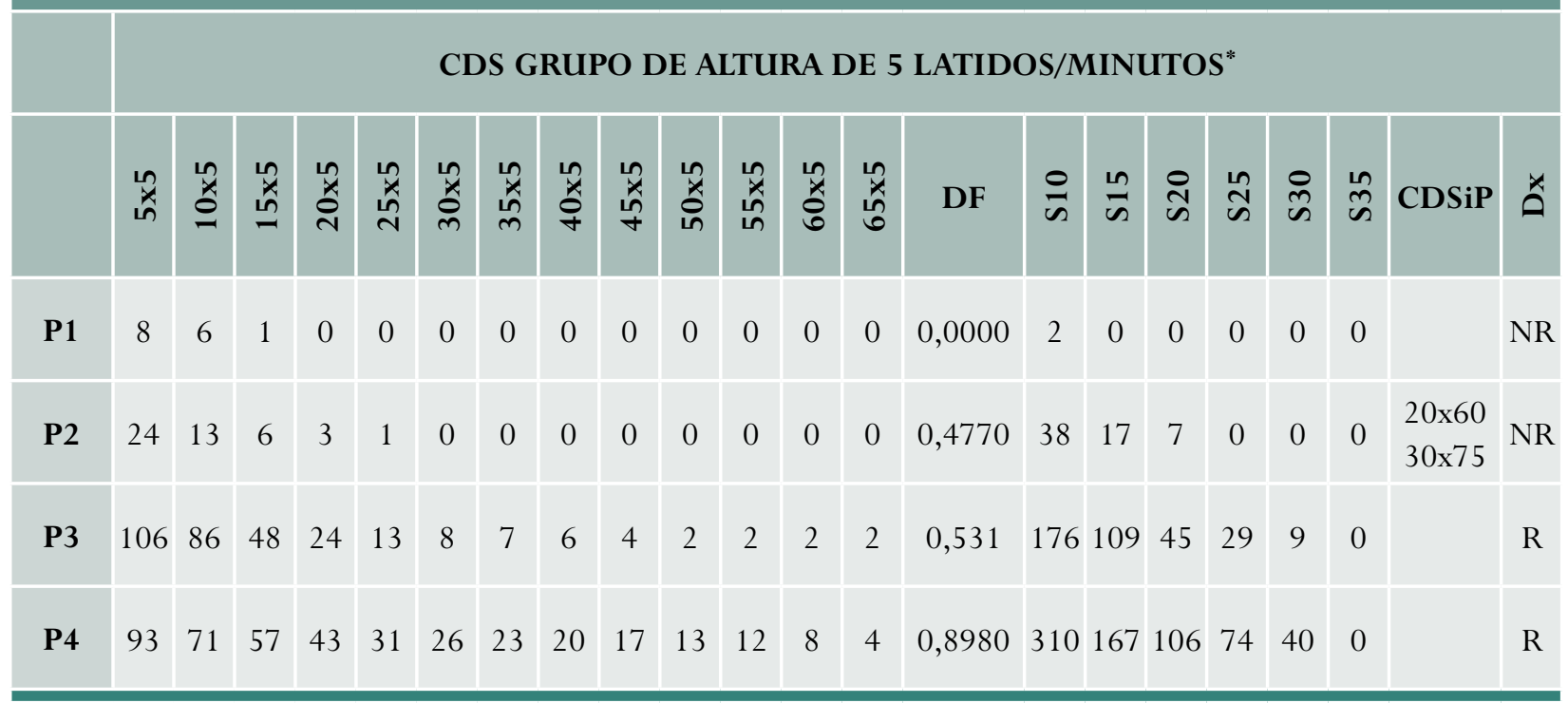

DF: Dimensión fractal. S10: Corresponde a la suma de los CDS del grupo de altura de 10 latidos/minuto, esta nomenclatura también se aplica a S15, S20, S25, S30, S35, que corresponden a la suma de los CDS de altura 15, 20, 25, 30 y 35 latidos/minuto respectivamente. Dx: Diagnóstico clínico convencional, NR: No reactiva, R: Reactiva 


\section{Tabla 2. Comparaciones con otras monitorías. DF asociada.}

\section{CDS GRUPO DE ALTURA DE 5 LATIDOS/MINUTO}

\begin{tabular}{|c|c|c|c|c|c|c|c|c|c|c|c|c|c|c|c|c|c|c|c|c|c|c|}
\hline No. & $\begin{array}{l}\text { Ln } \\
\text { î }\end{array}$ & 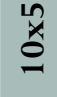 & $\begin{array}{l}\text { ñ } \\
\text { ñ }\end{array}$ & ¿̂̀ & ñ & ề & n̂t & ֻُ & $\begin{array}{l}L_{x}^{x} \\
10 \\
+\end{array}$ & ڤn & $\begin{array}{l}n \\
x \\
\text { nn } \\
\text { nn }\end{array}$ & 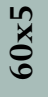 & 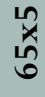 & DF & $\stackrel{0}{\text { n }}$ & $\frac{n}{n}$ & $\stackrel{\text { ิ }}{\text { ฟ }}$ & $\stackrel{\text { n}}{\sim}$ & હે & nn & CDSiP & $\stackrel{x}{a}$ \\
\hline 1 & 10 & 3 & 0 & 0 & 0 & 0 & 0 & 0 & 0 & 0 & 0 & 0 & 0 & 0,0000 & 2 & 0 & 0 & 0 & 0 & 0 & & NR \\
\hline 2 & 42 & 21 & 3 & 0 & 0 & 0 & 0 & 0 & 0 & 0 & 0 & 0 & 0 & 0,0000 & 21 & 10 & 3 & 0 & 0 & 0 & & NR \\
\hline 3 & 61 & 18 & 4 & 0 & 0 & 0 & 0 & 0 & 0 & 0 & 0 & 0 & 0 & 0,0000 & 37 & 22 & 0 & 0 & 0 & 0 & & NR \\
\hline 4 & 57 & 20 & 6 & 1 & 0 & 0 & 0 & 0 & 0 & 0 & 0 & 0 & 0 & 0,3070 & 44 & 3 & 0 & 0 & 0 & 0 & & NR \\
\hline 5 & 10 & 7 & 2 & 1 & 0 & 0 & 0 & 0 & 0 & 0 & 0 & 0 & 0 & 0,5017 & 11 & 2 & 0 & 0 & 0 & 0 & & NR \\
\hline 6 & 27 & 18 & 13 & 6 & 4 & 4 & 3 & 3 & 3 & 2 & 1 & 1 & 1 & 0,7153 & 59 & 40 & 30 & 0 & 0 & 0 & $\begin{array}{l}20 \times 40 \\
20 \times 50\end{array}$ & NR \\
\hline 7 & 25 & 15 & 7 & 2 & 1 & 1 & 0 & 0 & 0 & 0 & 0 & 0 & 0 & 0,4538 & 42 & 28 & 14 & 6 & 6 & 6 & $20 \times 50$ & $\mathrm{R}$ \\
\hline 8 & 17 & 16 & 15 & 11 & 9 & 8 & 6 & 3 & 2 & 1 & 1 & 0 & 0 & 0,7883 & 51 & 17 & 0 & 0 & 0 & 0 & & $\mathrm{R}$ \\
\hline 9 & 30 & 14 & 3 & 1 & 1 & 0 & 0 & 0 & 0 & 0 & 0 & 0 & 0 & 0,3777 & 8 & 5 & 5 & 0 & 0 & 0 & & NR \\
\hline 10 & 15 & 12 & 9 & 6 & 6 & 5 & 5 & 4 & 4 & 4 & 4 & 4 & 3 & 1,5848 & 23 & 8 & 0 & 0 & 0 & 0 & & NR \\
\hline
\end{tabular}

Las figuras 1 y 2 muestran un ejemplo del comportamiento hiperbólico observado en la distribución de frecuencias de aparición de CDS en una monitoría fetal particular y el análisis logarítmico que permite evaluar la dimensión fractal.

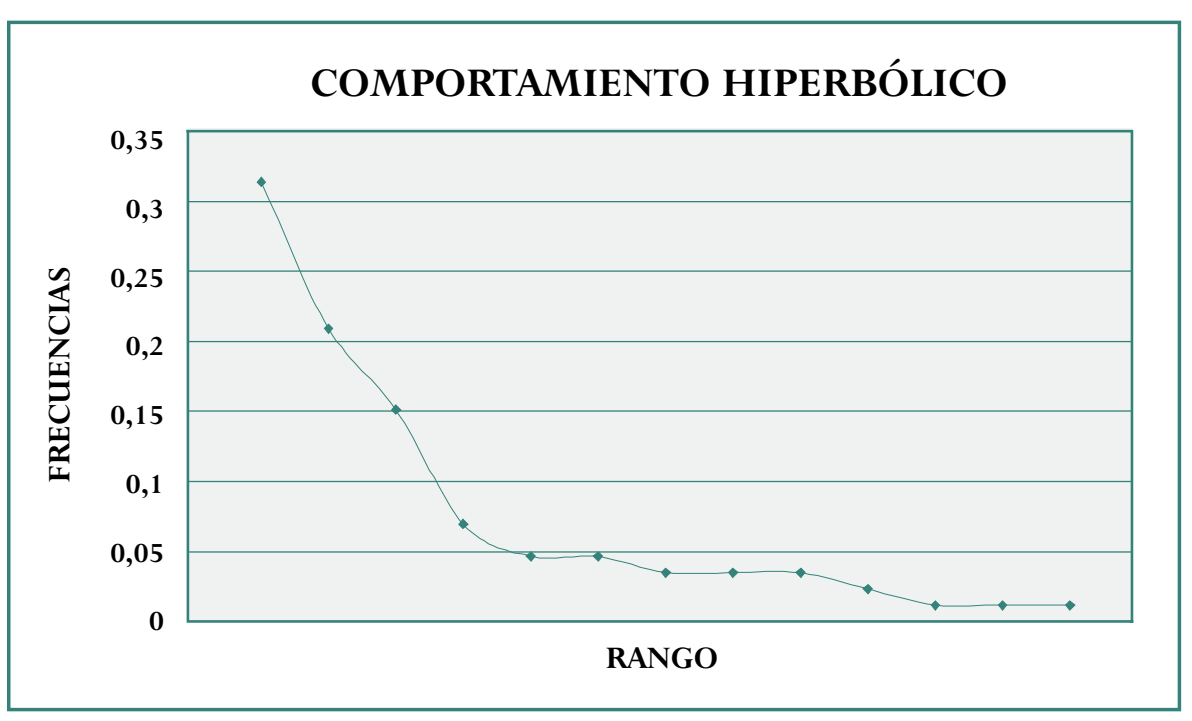

Figura 1. Distribución hiperbólica para la distribución de frecuencias de aparición de CDS de altura 5. Esta distribución corresponde a los valores obtenidos para una paciente particular.
Con los prototipos evaluados y la metodología se encontraron otros seis tipos de comportamientos simplificadores para cualquier monitoría, en los cuales aparecen las diferentes combinaciones de los parámetros obtenidos. Se pueden observar en la tabla 2:

En el número 2 se pierde la complejidad y aparecen CDS de 20, pero de muy bajo valor, en el número 6 aparecen CDS de 15 y 20 latidos/minuto y 2 CDSiP. También se presentan dos casos en los que no aparecen CDS de 20 y aparecen CDS de 15: en el número 8 la dimensión fractal es menor que uno y en el número 10 es mayor que uno. Además se presentan monitorías en las que aparecen CDS del grupo de 15 y de 20 latidos/minuto 


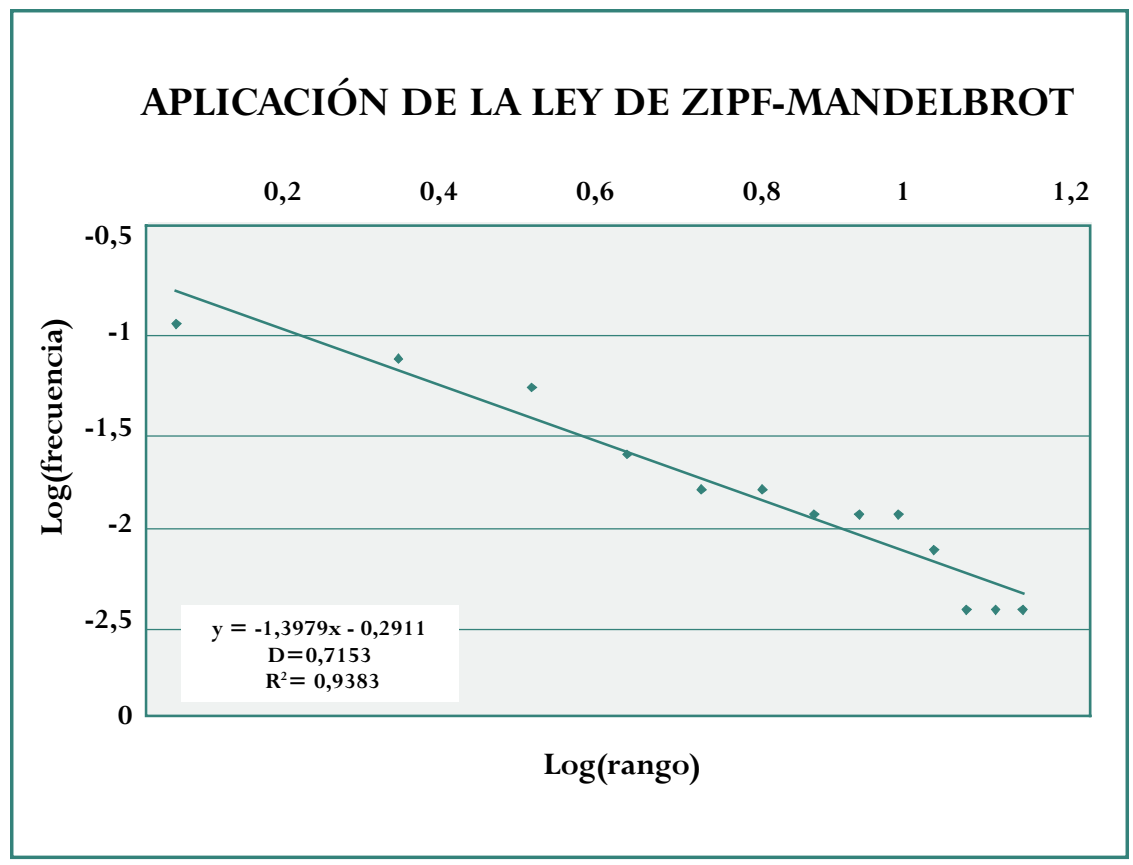

Figura 2. Aplicación de la ley de Zipf-Mandelbrot para la distribución de frecuencias de aparición de CDS de altura 5. Esta distribución corresponde a los valores obtenidos para una paciente particular del grupo A, su dimensión fractal es $\mathrm{D}=0,7153$ pretación de la concepción salud-enfermedad de los sistemas dinámicos basados en la comparación de aparición de todos los CDS posibles entre prototipos fundamentales y el resto de las monitorías. El método diagnóstico desarrollado evidencia una autoorganización matemática, jerárquica y estática para un feto sano, caracterizada por su grado de complejidad y la ausencia de CDSi pronunciados, la pérdida de complejidad o la presencia mínimo de un CDSiP, o un CDSi mayor o igual a $20 \mathrm{x} 40 \mathrm{y}$ menor o igual a 20x50 que este asociado simultáneamente a la presencia de un CDSi mayor o igual a 20x50, o la ausencia pero de bajo valor, el número 9; y finalmente, se tiene el número 7, con un CDSi no pronunciado y el resto de los parámetros asociados a la salud.

La monitoría dos y la sexta están enfermas, las monitorías ocho, nueve y diez en evolución a la enfermedad, hacia el prototipo uno y la siete es sana.

Los CDSiP que representan disminuciones pronunciadas en la frecuencia cardiaca fetal resultaron ser: 20x40, 20x50, 20x60, 30x75.

$\mathrm{Al}$ comparar el diagnóstico desarrollado con la metodología clínica convencional se encontró que una de cada dos monitorías del grupo sin factores de riesgo y uno de cada seis del grupo con factores de riesgo tiene un diagnóstico impreciso.

\section{DISCUSIÓN}

Este es el primer trabajo en el que se obtiene un diagnóstico matemático de la monitoría fetal a partir de la ley de Zipf-Mandelbrot y la reinter-

\footnotetext{
Esta monitoría pertenece al primogénito de los autores quinto y séptimo.
}

de CDS del grupo de 15 y/o de 20 latidos/minuto o combinaciones simultáneas de estas medidas diagnósticas son indicadores de enfermedad. Esta metodología general es preventiva y de aplicación clínica a cualquier caso particular.

Con la metodología matemática seguida, los parámetros de evaluación de la monitoría fetal se reducen a pocas medidas objetivas y reproducibles, que muestran una simplificación cuantitativa comparada con la evaluación clínica convencional y sus dificultades de subjetividad ${ }^{19,21-23}$ en la interpretación de la monitoría ${ }^{24}$ a partir de cinco parámetros que necesitan de 7 subparámetros particulares aclaratorios. Comparando los diagnósticos convencionales y el desarrollado para cada monitoría se encontró que para el grupo A son diferentes en el 16,3\% de los casos mientras que para el grupo B la diferencia entre los diagnósticos fue del $48 \%$ evidenciando que las diferencias entre los grupos no son tomadas en cuenta por la evaluación matemática.

Luego de la evaluación de los cuatro prototipos fundamentales se encontraron otros seis tipos de 
comportamientos simplificadores inferidos de los prototipos que junto con la metodología permitieron observar dinámicas asociadas a la enfermedad, la salud y a la evolución hacia la enfermedad o hacia la salud. Por ejemplo las monitorías ocho, nueve y diez de la tabla 2 diferencian qué tan comprometido está una monitoría comparado con el prototipo de enfermedad fundamental P1. Estas monitorías y otras evaluadas evidencian la capacidad de la metodología para discernir la variabilidad y su disminución en los monitorías, por lo que la no reactividad puede ser diferenciada y aclarada en este trabajo respecto al diagnóstico convencional. Con este trabajo estamos discerniendo la no reactividad de cualquier monitoría cuantificando su diferencia o igualdad respecto al prototipo P1, mostrando qué tan variable o no es una monitoría, lo que hace un diagnóstico preventivo en el que podemos discernir si hay evolución hacia la enfermedad, logrando hacer una cuantificación más fina de monitorías no reactivas y obviando esta denominación. Cuando una monitoría es reactiva también podemos discernir diferencias, pues unas tendrán CDSi no pronunciados, como en la monitoría siete de la tabla 2, monitoría obtenida durante el trabajo de parto y otros no los presentarán. Sin embargo, esta metodología cuantifica toda la posible variabilidad para cualquier monitoría obviando la nominación de reactividad o no del diagnóstico anterior.

Es importante aclarar que lo que refuerza la metodología diagnóstica es que se consideró un límite para las sumas de CDS de los grupos de altura de 20 y de 15 latidos por minuto, estableciendo para el primer grupo que valores menores a 14 de acuerdo a la evaluación de la monitoría siete de la tabla 2 , están evolucionando hacia la enfermedad al igual que para la sumatoria de CDS del grupo de altura 15 en donde veinte es considerada de bajo valor. Estos valores se pueden precisar más y son útiles en la evaluación de la evolución hacia la enfermedad, a menos que haya CDSiP o ausencia de la complejidad. La repetición de la monitoría en el tiempo basándose en estas medidas objetivas podría evidenciar qué tan comprometida o no está, por lo que estas medidas son de utilidad no sólo diagnóstica sino preventiva en la clínica, al inferir qué tan enfermos o no están los pacientes evaluados y su posible evolución a la enfermedad.

La aplicación de metodologías y concepciones físicas y matemáticas al estudio de los sistemas fisiológicos ha llevado a nuevas concepciones Salud-Enfermedad en la medicina, tal es el caso de Goldberger ${ }^{25}$ y Huikuri ${ }^{26}$ que lograron definir factores predictores de mortalidad en IAM en pacientes con fracción de eyección menor al 35\% con metodologías estadísticas a diferencia de esta generalización.

En un trabajo previo Rodríguezy colaboradores ${ }^{18}$ desarrollaron una generalización basada en la teoría de los sistemas dinámicos en la que caracterizaron monitorías fetales a partir de la teoría de la probabilidad con 4 CDS, constituyendo una primera aproximación muy simplificada comparada con la medición de todos los posibles CDS y los parámetros evaluados en este trabajo. Este trabajo se basa en una simplificación de la dinámica fisiológica cardiaca fetal, por lo que como en el trabajo anterior no se tienen en cuenta la enfermedad individuo madre/hijo, las contracciones uterinas, factor de riesgo o sin él, y es independiente de la edad gestacional; si es mayor de 28 semanas, independiente de poblaciones, de los factores de riesgo y útil para cada paciente.

A partir de investigaciones de la fisiología cardiaca basada en la teoría del caos, ${ }^{6,17,25,27,28}$ se ha planteado una nueva concepción de salud y enfermedad, en contra de la mirada homeostática tradicional. ${ }^{17}$ Basados en esta concepción y en las observaciones clínicas se hizo una reinterpretación del comportamiento de la frecuencia cardiaca fetal en el trazado de las monitorías escogiendo un registro prácticamente lineal y otro con desaceleraciones pronunciadas a cambio del registro aleatorio de la concepción de Goldberger, pues la enfermedad (en este caso el sufrimiento fetal agudo) no es aleatoria en el sentido de los sistemas dinámicos. Además 
se seleccionaron dos monitorías más con aceleraciones y variabilidad sin duda en su diagnóstico, asociadas a la salud. Esto unido a la aplicación de la ley Zipf-Mandelbrot y sus asociaciones con salud y enfermedad son el fundamento del diagnóstico realizado en este trabajo.

De acuerdo a la ley de los lenguajes naturales ${ }^{9-12}$ una dimensión fractal tendiendo a cero o mayor a 1 indica la pérdida de la coherencia del texto. Si se ponen palabras en otros idiomas al azar, la dimensión fractal indica la pérdida de coherencia del texto. En el caso del sistema inmune ${ }^{13,14}$ los ratones atímicos e irradiados corresponden a la dimensión fractal hacia 0 y 1 respectivamente. En este trabajo, una dimensión fractal hacia 0 muestra cómo se pierde la complejidad como en trabajos pasados ${ }^{10-14}$ por lo que la pérdida de complejidad, presencia de tres o menos tipos de CDS se asociaron a la enfermedad. Respecto a valores en la dimensión fractal mayor que uno, en los lenguajes naturales significa pérdida de coherencia, pero en nuestro caso no necesariamente, pues encontramos repeticiones del mismo valor en varios CDS consecutivos lo que hace que el valor de la dimensión fractal resulte fuera de cero y uno, pero lo que nos dice es que hay una mayor variabilidad de presentación de CDS y no que haya enfermedad.

La medicina actual basada en la epidemiología y la estadística estudia causas y efectos de la salud y la enfermedad. Con el desarrollo de la mecánica estadística, ${ }^{29}$ la mecánica cuántica ${ }^{30}$ y la teoría del $\operatorname{caos}^{31,32}$ los fundamentos de la física moderna y de la ciencia en general dejaron de ser causales, lo que en palabras de Schrödinger ${ }^{33}$ respecto a las causas: "quita su base racional a aquella manera de pensar contraída en la costumbre". En este trabajo como en la física moderna no tenemos causas ni consecuencias sino órdenes matemáticos acausales asociados con la salud y la enfermedad basados en la experiencia clínica. En este sentido este no es un trabajo matemático aplicado a la medicina sino un trabajo físico y matemático de aplicación clínica. Desde la perspectiva del caos, el antes y el después de cada valor del latido cardiaco es impredecible, como en otros sistemas dinámicos ${ }^{34-36}$ y en el caso específico del caos fetal ${ }^{37}$ esta impredecibilidad afecta sensiblemente las evaluaciones clínicas perinatales antes o después del nacimiento. En este trabajo las asociaciones salud - enfermedad no son antes y después en el tiempo sino simultáneamente evitando los problemas del caos respecto a la impredecibilidad, además de no realizar contrastaciones perinatales por la metodología acausal basada en asociaciones salud-enfermedad.

Este trabajo presenta una nueva metodología de análisis de la monitoría fetal, basada en concepciones físicas y matemáticas. Se ha logrado una generalización teórico-práctica preventiva para la dinámica cardiaca fetal con la que ha sido posible definir un diagnóstico objetivo y reproducible de aplicación a cada caso particular.

\section{Limitaciones}

En este estudio sólo se toman en cuenta los CDSi pronunciados, pero no se realiza un análisis completo respecto a la totalidad de CDSi, sin que esto invalide la generalización y aplicación de la metodología diagnóstica desarrollada. Pues los CDSiP son los CDS que caracterizan objetivamente tanto el sufrimiento fetal agudo como las disminuciones de la frecuencia cardiaca fetal determinantes para diferenciar salud de enfermedad.

\section{Aplicaciones futuras}

Se requieren monitorías tomadas en diferentes tiempos durante la gestación a una misma paciente para corroborar la precisión de las medidas de evolución salud - enfermedad y para confirmar la metodología diagnóstica y precisar las relaciones para monitorías fetales de gemelos.

\section{CONCLUSIONES}

1. El diagnóstico matemático desarrollado permite evaluar el estado de salud o enfermedad del feto. 
La dimensión fractal mayor que cero de la distribución de CDS en cualquier monitoría fetal y la ausencia de CDSi es asociada a la salud. La ausencia de complejidad o la presencia mínimo de un CDSiP o la presencia de un CDSi mayor o igual a 20x50 asociada a la de un CDSi que este entre 20x40 y 20x50, o la ausencia de CDS del grupo de altura 15 y/o de 20 latidos/minuto o combinaciones simultáneas de estas medidas diagnósticas son indicadores de enfermedad.

2. Esta metodología generalista es útil para cualquier caso particular y muestra que la evaluación clínica convencional podría fallar en una de cada dos monitorías del grupo sin factores de riesgo (48\%) y en una de cada seis monitorías del grupo con factores de riesgo (16,3\%); sin embargo, la metodología diagnóstica es independiente de muestras poblacionales y de la presencia o ausencia de factores de riesgo.

\section{DEDICATORIA}

En una ocasión se llevaba a cabo el recital de un gran pianista, una vez acabado el recital, uno de los espectadores se acercó al pianista y le dijo:

- Yo hubiera dado la vida por haber tocado así - y el pianista le contestó:

- Yo la dí.

El grupo INSIGHT dedica esta sinfonía a los niños de Colombia y del mundo.

\section{AGRADECIMIENTOS}

Agradecemos a todos los MAESTROS en especial al maestro Maharishi Mahesh Yogui y al grupo DHARMA que iluminó nuestro camino y el de muchos por venir.

Agradecemos al Instituto Colombiano para el Desarrollo de la Ciencia y la Tecnología "Francisco José de Caldas" - COLCIENCIAS - la financiación de este proyecto registrado con código No. 11010416380, aprobado en la convocatoria Salud - 2004 por su apoyo a nuestro grupo de investigación.
Al Dr. Jaime Gallego, decano de la facultad de medicina, por su apoyo incondicional, a la unidad administrativa de la facultad de medicina por su ayuda y eficacia en los procesos institucionales necesarios para llevar a cabo esta investigación, al Dr. Rubén Caicedo y a Ingrid Prieto por su apoyo en el camino para concretar los objetivos del grupo Insight.

Agradecemos al Instituto Materno Infantil en especial al director Dr. Odilio Méndez por su lucha para que el hospital no fuera cerrado, como también a la jefe Patricia Farias por su apoyo al instituto en los momentos más difíciles.

Agradecemos a los doctores Alberto Páez y Carolina Cifuentes por su sensibilidad y por la consecución de Historias Clínicas y de las monitorías fetales.

\section{REFERENCIAS}

1. Mandelbrot B. The Fractal Geometry of Nature. San Francisco: W.H. Freeman; 1982. p. 341-48.

2. Mandelbrot B. Introducción. En: Los Objetos Fractales. Barcelona: Tusquets Editores S.A.; 2000. p. 13-26.

3. Mandelbrot B. ¿Cuánto mide la costa de Bretaña? En: Los Objetos Fractales. Barcelona: Tusquets Editores S.A.; 2000. p. 27-50.

4. Peitgen H, Jurgens H, Saupe D. Limits and self similarity. En: Chaos and Fractals. New Frontiers of Science. New York: Springer Verlag; 1992. p. 135-82.

5. Peitgen H, Jurgens H, Saupe D. Lenght, area and dimension. Measuring complexity and scalling properties. En: Chaos and Fractals. New Frontiers of Science. New York: Springer Verlag; 1992. p. 183-228.

6. Goldberger AL, Rigney DR, West BJ. Chaos and fractals in human physiology. Sci Am 1990;262:42-9.

7. Baish JW, Jain RK. Fractals and cancer. Cancer Res 2000;60:3683-8.

8. Rodríguez J, Mariño M, Avilán N, Echeverri D. Medidas fractales de arterias coronarias en un modelo experimental de reestenosis. Armonía matemática intrínseca de la estructura arterial. Rev Colomb Cardiol 2002;10:65-72.

9. Zipf GK. Human Behavior and the Principle of Least Effort. Cambridge, M.A.: Addison-Wesley Press; 1949. 
10. Mandelbrot B. Cambios de escala y leyes potenciales sin geometría. En: The Fractal Geometry of Nature. San Francisco: W.H. Freeman; 1982. p. 477-87

11. Mandelbrot B. Árboles jerárquicos o de clasificación, y la dimensión. En: Los Objetos Fractales. Barcelona: Tusquets Editores S.A.; 2000. p. 161-66.

12. Mandelbrot, B. Structure formelle des textes et comunication. Word 1954;10:1-27.

13. Burgos JD, Moreno-Tovar P. Zipf-scaling behavior in the immune system. Biosystems 1996;39:227-32.

14. Burgos JD. Fractal representation of the immune B cell repertoire. Biosystems 1996;39:19-24.

15. Rodríguez J. Comportamiento fractal del repertorio T específico contra el alergeno Poa P9. Rev Fact Med Univ Nac Colomb 2005;53:72-8.

16. Devaney RL. A first course in chaotic dynamical systems; theory and experiments. Reading Mass: Addison Wesley; 1992.

17. Goldberger AL, Amaral LA, Hausdorff JM, Ivanov PCh, Peng CK, Stanley HE. Fractal dynamics in physiology: alterations with disease and aging. Proc Natl Acad Sci USA 2002; 99 Suppl 1:2466-72.

18. Rodríguez J, Carmona V, Avilán N, Hincapié P, Análisis de la monitoría fetal con la teoría de la probabilidad. Rev Colomb Obstet Ginecol 2004;55:267-78.

19. Borgatta L, Shrout PE, Divon MY. Reliability and reproducibility of nonstress test readings. Am J Obstet Gynecol 1988;159:554-8.

20. Mood AM, Graybill FA, Boes D. Introduction to the theory of statistics. 3a. ed. Singapore: McGraw-Hill; 1974. p. 482-88.

21. Benson R. Diagnóstico y tratamiento ginecoobstétrico. 4 ed. México DF: El Manual Moderno SA de CV; 1986.

22. Sánchez F. Alto riesgo obstétrico. Bogotá: Universidad Nacional de Colombia; 1988.

23. Pritchard JA, MacDonald PC, Gant NF (eds). Williams Obstetricia. Barcelona: Salvat Editores; 1986.

24. National Institute of Child Health and Human Development Research Planning Workshop. Electronic fetal heart rate monitoring: research guidelines for interpretation. Am J Obstet Gynecol 1997;177:1385-90.

25. Goldberger AL, West BJ. Fractals in physiology and medicine. Yale J Biol Med 1987;60:421-35.

26. Huikuri HV, Makikallio TH, Peng CK, Goldberger AL, Hintze U, Moller M. Fractal correlation properties of R-R interval dynamics and mortality in patients with depressed left ventricular function after an acute myocardial infarction. Circulation 2000;4:47-53.

27. Goldberger AL. Non-linear dynamics for clinicians: chaos theory, fractals, and complexity at the bedside. Lancet 1996;347:1312-4.

28. Lipsitz LA, Goldberger AL. Loss of 'complexity' and aging. Potential applications of fractals and chaos theory to senescence. JAMA 1992;267:1806-9.

29. Feynman R. Los principios de la mecanica estadistica. En: Física. Vol 1, Cap 40. Addison-Wesley Iberoamericana S.A.; 1987.

30. Feynman R. Comportamiento cuántico. En: Física. Vol 1, Cap 37. Addison-Wesley Iberoamericana S.A.; 1987.

31. Fernández-Rañada A. Movimiento caótico. En: Orden y Caos. Scientific American. Prensa Científica S.A.; 1990. p. $66,77$.

32. Crutchfield J, Farmer D, Packard N, Shaw R. Caos. En: Orden y Caos. Scientific American. Prensa Científica S.A.; 1990. p. 78-90.

33. Schrödinger E. ¿Qué es una ley física? Rev Cultural de Occidente 1968;11:375-84.

34. Procaccia I. Universal properties of dynamically complex systems: the organization of chaos. Nature 1988;333:618-23.

35. Kloeden PE, Mees AI. Chaotic phenomena. Bull Math Biol 1985;47:697-738.

36. Denton TA, Diamond GA, Helfant RH, Khan S, Karagueuzian H. Fascinating rhythm: a primer on chaos theory and its application to cardiology. Am Heart J 1990;120:1419-40.

37. Gough NA. Fractals, chaos, and fetal heart rate. Lancet 1992;339:182-3. 